\title{
THE IN VITRO AND IN VIVO EFFECT OF SULFONAMIDES UPON THE STREPTOCOCCAL ANTIFIBRINOLYSIN TEST
}

\author{
By WILLIAM M. M. KIRBY AND LOWELL A. RANTZ \\ (From the Department of Medicine, Stanford University School of Medicine, San Francisco)
}

(Received for publication January 6, 1942)

The streptococcal antifibrinolysin test of Tillett and Garner (1) was at first thought to be diagnostic of recent hemolytic streptococcal infections. Subsequent studies have shown, however, that the test may be non-specific, increased amounts of antifibrinolysin having been noted in other diseases, such as gonococcal arthritis (2) streptococcus viridans bacteremia (3) and pneumococcal pneumonia (4).

The present communication deals with the relation of sulfonamides to the production of antifibrinolysin. An increased resistance to fibrinolysis was found by Hines, Hoover, and Graff (5) in patients receiving sulfanilamide. This increased liquefaction time paralleled, in general, the rise or fall of the concentration of sulfanilamide in the blood stream, and the fibrinolysin time returned to its normal level in from two to five days after administration of the drug had been discontinued. From these results it seemed evident that sulfanilamide in some manner increases the antifibrinolytic properties of the tissues of the host, and it was suggested that this action may aid the bacteriostatic properties of the drug by preventing the spread of organisms throughout the body.

Since sulfonamides are used so widely at the present time, especially in diseases in which the antifibrinolysin test is of the greatest interest, it seemed worth while to attempt to confirm these results.

\section{PROCEDURE AND RESULTS}

Three strains of hemolytic streptococci were used as sources of fibrinolysin:

Number 1, a solid lytic substance, prepared from a Lancefield group A organism isolated from a human infection. The method was that of Garner and Tillett (6), and fresh solutions, containing $5 \mathrm{mgm}$. per $0.5 \mathrm{cc}$. were prepared shortly before performing each test.

Number 2, another Lancefield group A strain, selected especially because it produced considerably less fibrinolysin than the one used in making the solid substance. This was transferred daily in veal infusion broth:
Number 3, a Lancefield group C strain, isolated from the blood stream of a bacteremic patient, which was also transferred daily in veal infusion broth.

Numbers 1 and 3 regularly lysed normal human fibrin clots in twenty minutes or less. With Number 2, lysis usually occurred in two to four hours. The fibrin clots of patients with recent hemolytic streptococcal infections showed partial or complete resistance to liquefaction with all three lytic strains.

The antifibrinolysin tests were performed according to the method of Tillett and Garner (1), except that the plasma fibrinolysin solution was incubated for fifteen minutes before adding $\mathrm{CaCl}_{2}$.

\section{Experiment 1}

Studies were made of the in vitro effect of sulfanilamide. Antifibrinolysin tests were performed in duplicate on the plasmas of seven normal individuals. One tube contained no sulfanilamide, while the other contained the drug in a concentration of $20 \mathrm{mgm}$. per cent. Only the solid lytic substance was used in this experiment.

Results. The liquefaction times varied from five to twenty-five minutes. The time required for lysis in the tubes containing sulfanilamide was in every instance almost identical with that in the tubes to which no drug had been added. These results are in agreement with those of Huntington (7), who also found that the addition of sulfanilamide in vitro produces no effect upon the antifibrinolysin test.

\section{Experiment 2}

Antifibrinolysin tests were performed on six children with scarlet fever who were treated with full therapeutic doses of sulfanilamide or sulfathiazole. The tests were performed once or twice in the four or five days during which the drugs were administered. Again, only the solid lytic substance described above was used for the tests. 
These patients were followed for several weeks after the drugs had been discontinued, antifibrinolysin tests being performed at weekly intervals.

Results. The liquefaction times during the period of sulfonamide administration were under twenty minutes in all instances. These determinations were made during the first five or six days of the disease, before an increase had occurred in the antifibrinolysin titer due to the streptococcal infection per se. Such an increase in the amount of antifibrinolysin did develop later in four of the six children, beginning during the latter part of the second week of the disease. In two, lysis occurred after several hours, while in the other two there was complete resistance to lysis at the end of twenty-four hours. Thus, it is evident that sulfonamide administration caused no increase in antifibrinolysin in these individuals who later developed high titers as a result of hemolytic streptococcal infection.

\section{Experiment 3}

Six patients on the medical wards, mostly diagnostic problems with no demonstrable organic disease, were selected. All had normal temperatures and leukocyte counts. Antifibrinolysin tests were performed on two successive days before the administration of sulfonamides in order to establish a constant base line. The three lytic substances previously described were used in all the tests. The patients were then given sulfonamides orally, an initial dose of 4 grams followed by 1 gram every four hours. Blood was collected at twenty-four hour intervals after the initial dose for antifibrinolysin tests and for determinations of sulfonamide blood levels which were made according to the method of Bratton and Marshall (8). Of the six patients, two received sulfanilamide, two sulfathiazole, and two sulfadiazine. In all instances the antifibrinolysin tests were begun within four hours after the plasma was collected.

Results. The accompanying figure shows the results in all six patients. It can be readily seen that administration of the sulfonamides had no effect whatsoever upon the results of the antifibrinolysin tests. Blood levels of the magnitude desirable for therapy of severe infections were attained in most instances. Patient 6 received sulfadiazine for two weeks, during which the fibrinolysin times were the same as those shown on the chart.

\section{COM MENT}

Evidence is presented to show that sulfonamides have no effect whatsoever on the streptococcal antifibrinolysin test, either in vitro or in vivo. These results are not in accord with those of Hines, Hoover, and Graff (5) who found that, in vivo, sulfonamide administration caused an increased resistance to fibrinolysis.

The following explanation may account for these conflicting results: Massell, Mote, and Jones (9), in studying the quantitative aspects of the antifibrinolysin test, showed that, when the concentration of fibrinolysin is low, a very slight decrease in concentration will produce marked prolongation of the liquefaction time. For example, a liquefaction time of four hours can be easily increased to twenty-four hours by decreasing only slightly the concentration of fibrinolysin. Such a weak lytic strain was apparently used in the experiments of Hines et al., for the initial liquefaction time was relatively long in every instance. Indeed, in spite of the statement that those with initial liquefaction times of over four hours were excluded, it can be seen from their protocols that two were in the neighborhood of five hours. Thus, any factor associated with sulfonamide administration which would only slightly decrease the fibrinolysin concentration would cause an apparent increased resistance to fibrinolysis. One such factor might be the inhibiting effect of the sulfanilamide in the patient's plasma upon the bacterial growth of the broth culture during the three or four hours required to perform the test. This might decrease the amount of fibrinolysin sufficiently to prolong markedly the liquefaction time. This would, of course, be an in vitro rather than an in vivo effect. The in vitro experiments of Huntington (7), and also those described here, were performed with more actively lytic strains, so that the results are not comparable. This explanation, if correct, is dependent upon a very weak lytic substance, since the amount of sulfonamide in the patient's plasma is small.

The Lancefield group C strain was actively lytic, comparable to the solid preparation of an active group A strain. Further studies of the immuno- 

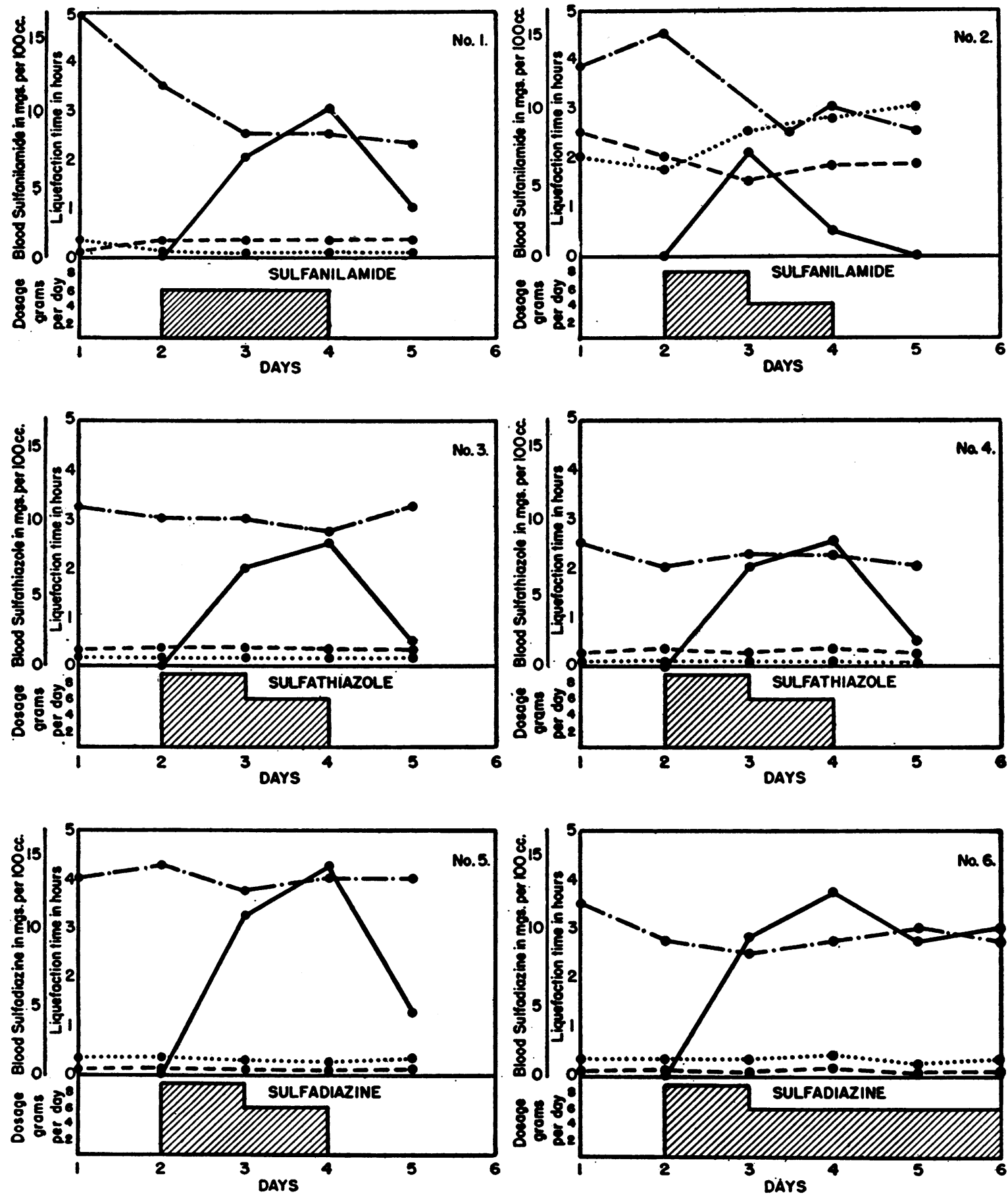

THIS FIGURE SHOWS THE RESULTS IN SIX PATIENTS, OF WHICH TWO RECEIVED SULFANILAMIDE; TWO SULFATHIAZOLE, AND TWO SULFADIAZINE. SOLID. LINE REPRESENTS BLOOD LEVELS. LYTIC STRAINS ARE AS FOLLOWS; NO.

NO. 2.

Fig. 1. Failure of Sulfonamide Administration to Effect the Streptococcal Antifibrinolysin Test in Vivo 
logical properties of this organism will be presented elsewhere. The scarlet fever cases were of special interest. In them sulfonamide administration had no effect upon fibrinolysis but they later developed increased amounts of antifibrinolysin as a result of the hemolytic streptococcal infection.

\section{SUM MARY AND CONCLUSIONS}

Evidence is presented to show that sulfonamide administration, both in vitro and in vivo, has no effect upon the streptococcal antifibrinolysin test. The results of the in vivo experiments are not in agreement with those reported from another laboratory. The reasons for this discrepancy are discussed.

\section{BIBLIOGRAPHY}

1. Tillett, W. S., and Garner, R. L., The fibrinolytic activity of hemolytic streptococci. J. Exper. Med., 1933, 58, 485.

2. Myers, W. K., Keefer, C. S., and Holmes, W. F., Jr., The resistance to fibrinolytic activity of the hemo- lytic streptococcus with special reference to patients with rheumatic fever and rheumatoid (atrophic) arthritis. J. Clin. Invest., 1935, 14, 119.

3. Waaler, E., Development of antifibrinolytic properties in blood of patients with rheumatic fever, chronic infective arthritis, and bacterial endocarditis. $J$. Clin. Invest., 1937, 16, 145.

4. Boisvert, P. L., The streptococcal antifibrinolysin test in clinical use. J. Clin. Invest., 1940, 19, 65.

5. Hines, L. E., Hoover, A. H., and Graff, E., Effect of sulfanilamide on fibrinolytic activity of hemolytic streptococci. Arch. Int. Med., 1940, 65, 744.

6. Garner, R. L., and Tillett, W. S., Biochemical studies on the fibrinolytic activity of hemolytic streptococci. 1. Isolation and characterization of fibrinolysin. J. Exper. Med., 1934, 60, 239.

7. Huntington, R. W., Failure of sulphanilamide to prevent hemolysis, fibrinolysis, and production of erythrogenic toxin by hemolytic streptococci in vitro. Proc. Soc. Exper. Biol. and Med., 1938, 38, 328.

8. Bratton, A. C., and Marshall, E. K., Jr., A new coupling component for sulfanilamide determination. J. Biol. Chem., 1939, 128, 537.

9. Massell, B. F., Mote, J. R., and Jones, T. D., The quantitative relation of fibrinolysin and antifibrinolysin. J. Immunol., 1939, 36, 45. 